\title{
Correlation between serum Hcy level and severity of chronic HBV hepatitis
}

https://doi.org/10.1515/pteridines-2019-0007

received January 21, 2019; accepted March 4, 2019.

Abstract: Objective The aim of this work was to investigate the serum homocysteine (Hcy) level and severity of hepatitis B virus (HBV).

Methods 72 patients with chronic HBV and 28 healthy controls were included in this work. Of the included 72 chronic HBV hepatitis patients, 20 patients had mild disease, 31 had moderate disease and 21 had severe disease. The serum homocysteine (Hcy), alanine aminotransferase (ALT), aspartate aminotransferase (AST) and total bilirubin (TBIL) were examined in both HBV hepatitis and control patients.

Results Significant statistical difference was observed for serum Hcy, ALT, AST and TBIL in different groups $(p<0.05)$. The serum Hcy, ALT, AST and TBIL in the severe group were significantly higher than those of other groups with statistical difference $(\mathrm{p}<0.05)$; significant correlation was observed between serum Hcy and AST in the moderate $(\mathrm{r}=0.43, \mathrm{p}<0.05)$ and severe disease groups $(\mathrm{r}=0.63$, $\mathrm{p}<0.05)$. However, the correlation between Hcy and ALT, and Hcy andTBIL were not statistically significant in any group ( $p>0.05)$.

Conclusion The serum Hcy level in patients with hepatitis $\mathrm{B}$ reflects the damage to the of liver. The continuous increase of serum Hcy level can be regarded as a risk factor for the progression of hepatitis, and it can be used as serological marker for clinical diagnosis, treatment and prognosis.

Keywords: hepatitis B virus; homocysteine; severity; ALT; AST.

\section{Introduction}

Hepatitis B is a disease caused by hepatitis B virus (HBV) infection, which mainly causes inflammation and multiple organ damage $[1,2]$. According to World Health Organization (WHO) epidemiological data, about 200 million people worldwide have been infected with HBV, and about 600,000 people die every year from HBV-related diseases [3]. China has a heavy burden of HBV infection. Epidemiological studies show that the number of HBsAg positive carriers in China is over 100 million $[4,5]$.

The liver is an important organ of amino acid metabolism. Damage to the liver induces the disorder of Hcy metabolism and can further hyprehomocysteinemia. Moreover, Hcy accelerates the progression of liver disease by oxidative stress, therefore resulting in damage to hepatic cells. When the hepatitis $B$ virus invades the body and causes liver damage, it will lead to the disorder of amino acid metabolism and change in the serum amino acid spectrum. Hcy is a non-proteinogenic sulfurcontaining $\alpha$-amino acid biosynthesised via methionine demethylation [6].

The main metabolic organ of Hcy is the liver. With the gradual aggravation of liver tissue damage in HBV patients, the activity of methionine metabolic enzymes decreases due to parenchymal cell damage and the plasma Hcy level increases gradually [7]. The elevated serum Hcy increases the oxidative stress on the liver and leads to the injury of hepatic cells [8]. Therefore, serum Hcy can be used as a biochemical marker to reflect the degree of liver injury in patients with Hepatitis B.

\section{Materials and methods}

\section{Patients and controls}

Zhuji People‘s Hospital of Zhejiang Province 311800 PR China, E-mail:723402475@qq.com

Meiping Zhao: Department of infectious diseases, the People's Hospital of Fenghua Ningbo, Zhejiang Province 315500 PR China Xinjun Wang: Department of Dermatology, the People's Hospital of Fenghua Ningbo, Zhejiang Province 315500 PR China
72 patients with chronic Hepatitis B and 28 healthy controls were included in this study. Of the included 72 chronic HBV hepatitis patients, 20 had mild disease, 31 had moderate disease and 21 had severe disease. There 
Table 1: Serum Hcy, ALT, AST and TBIL level in different groups $(\bar{x} \pm s)$.

\begin{tabular}{llllll}
\hline Groups & Hcy $(\mu \mathrm{mol} / \mathrm{L})$ & ALT $(\mathrm{U} / \mathrm{L})$ & AST $(\mathrm{U} / \mathrm{L})$ & TBIL $(\mu \mathrm{mol} / \mathrm{L})$ & Serum creatinine $(\mu \mathrm{mol} / \mathrm{L})$ \\
\hline Control $(\mathrm{n}=28)$ & $8.82 \pm 3.17$ & $21.52 \pm 10.96$ & $19.78 \pm 10.22$ & $9.62 \pm 4.06$ & $82.12 \pm 8.26$ \\
HBV hepatitis & & & & & \\
Mild (20) & $13.92 \pm 6.52$ & $168.50 \pm 49.57$ & $145.80 \pm 44.96$ & $143.60 \pm 49.80$ & $86.53 \pm 9.20$ \\
Moderate (31) & $22.06 \pm 7.45$ & $209.80 \pm 50.80$ & $182.90 \pm 57.11$ & $165.50 \pm 63.82$ & $79.6 \pm 8.42$ \\
Severe (21) & $29.97 \pm 12.24$ & $315.50 \pm 73.98$ & $274.00 \pm 95.94$ & $245.80 \pm 91.08$ & $88.4 \pm 7.96$ \\
\hline
\end{tabular}

were 49 men and 23 women with the mean age of $45.2 \pm$ 12.6 and a mean course of disease of $7.02 \pm 2.64$ years. The mean age of the healthy controls was $42.7 \pm 8.6$ with 17 males and 11 females.

Ethical approval: The research related to human use has been complied with all the relevant national regulations, institutional policies and in accordance the tenets of the Helsinki Declaration, and has been approved by the Medical Ethics Committee of People's Hospital of Fenghua District.

Informed consent: Informed consent has been obtained from all individuals included in this study

\section{Serum Hcy, ALT, AST and TBIL examination}

$5 \mathrm{ml}$ peripheral venous blood was obtained from patients and healthy controls and was centrifuged at $3000 \mathrm{r} / \mathrm{min}$ for 15 minutes to obtain the serum and was stored in the refrigerator at $5^{\circ} \mathrm{C}$ for the next use. Serum Hcy, ALT, AST and TBIL were measured by automatic biochemical analyzer (SIEMENS ADVIA 2400).

\section{Statistical analysis}

Spss17.0 software (SPSS, Inc., Chicago, IL, USA) was used for data analysis. Prism 7.0 (http://www.graphpad. $\mathrm{com} / \mathrm{scientific-software/prism/)} \mathrm{software} \mathrm{was} \mathrm{used} \mathrm{to}$ draw the statistical figures. The measurement data was expressed by $\bar{x} \pm s$ and compared between groups by $\mathrm{F}$ test. Correlation between Hcy and ALT, AST, TBIL was analyzed by Pearson correlation test. Two tails $\mathrm{P}<0.05$ was deemed as a statistically significant difference.

\section{Results}

\section{Serum Hcy, ALT, AST and TBIL levels in different groups}

The serum Hcy, ALT, AST and TBIL levels of the control, and HBV hepatitis mild, moderate and severe groups are shown in Table 1. Significant statistical difference was observed for serum Hcy, ALT, AST and TBIL in different groups ( $p<0.05$ ). The serum Hcy, ALT, AST and TBIL in the severe group were significantly higher than those of other groups with statistical difference $(\mathrm{p}<0.05)$, Figure 1.

\section{Correlation of serum ALT, AST, TBIL and Hcy}

The correlation between ALT, AST, TBIL and Hcy were analyzed by Pearson correlation test. Significant correlation was observed between serum Hcy and AST in the moderate $(\mathrm{r}=0.43, \mathrm{p}<0.05)$ and severe disease groups $(r=0.63, p<0.05)$, Table 2. However, the correlation between Hcy and ALT, TBIL were not statistically significant in any group ( $p>0.05$ ), Figure 2.

\section{Discussion}

The pathogenesis of hepatitis B is an inflammation mediated by immune over-expression, mainly the imbalance between Thl/Th2 [9, 10]. The damage of hepatic tissueis due to theimmune system being sensitized by HBV, which stimulates the immune system, participates in host immune defense response, and produces corresponding antibodies and/or cellular non-specific immune response [11-14]. The immune system not only clears HBV but also attacks hepatic cells with the HBV antigen on their surface, which is the main cause of apoptosis of hepatic cells and inflammation of liver tissue. The liver is an important organ of Hcy metabolism. Hepatitis B and other 
Table 2: Correlation between Hcy and ALT, AST, TBIL.

\begin{tabular}{lllll}
\hline Correlation & $\mathrm{r}$ & $\mathbf{9 5 \% \mathrm { Cl }}$ & $\mathbf{P}$ & Equation \\
\hline Hcy and ALT & & & \\
Mild disease & 0.18 & $-0.28 \sim 0.58$ & 0.45 & $\mathrm{Y}=1.431^{\star} \mathrm{X}+148.5$ \\
Moderate disease & 0.22 & $-0.14 \sim 0.54$ & 0.23 & $\mathrm{Y}=1.467^{\star} \mathrm{X}+177.5$ \\
Severe disease & 0.21 & $-0.24 \sim 0.59$ & 0.36 & $\mathrm{Y}=1.27^{\star} \mathrm{X}+277.4$ \\
Hcy and AST & & & & $\mathrm{Y}=2.95^{\star} \mathrm{X}+104.7$ \\
Mild disease & 0.41 & $-0.04 \sim 0.72$ & 0.07 & $\mathrm{Y}=3.169^{\star} \mathrm{X}+113.0$ \\
Moderate disease & 0.43 & $0.09 \sim 0.68$ & 0.02 & $\mathrm{Y}=4.929^{\star} \mathrm{X}+126.2$ \\
Severe disease & 0.63 & $0.27 \sim 0.83$ & $<0.01$ & $\mathrm{Y}=2.959^{\star} \mathrm{X}+102.5$ \\
Hcy and TBIL & & & & $\mathrm{Y}=2.299^{\star} \mathrm{X}+115.3$ \\
Mild disease & 0.37 & $-0.09 \sim 0.70$ & 0.11 & $\mathrm{Y}=0.8326^{\star} \mathrm{X}+220.8$ \\
Moderate disease & 0.28 & $-0.08 \sim 0.58$ & 0.12 & 0.63 \\
Severe disease & 0.11 & $-0.34 \sim 1.52$ & & \\
\hline
\end{tabular}

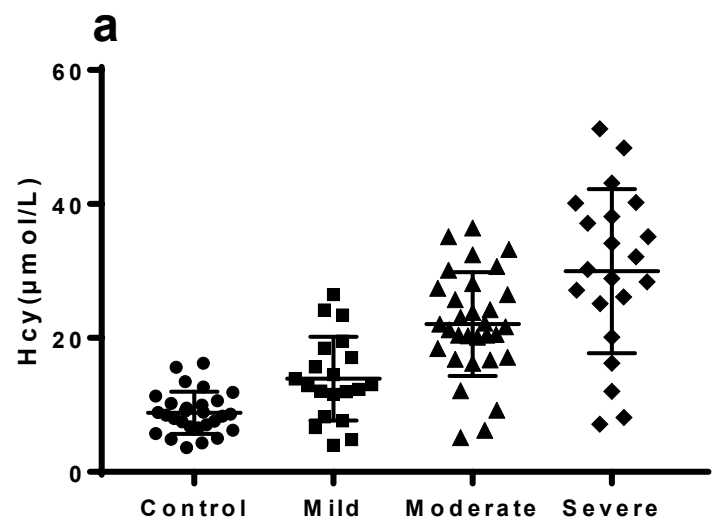

C

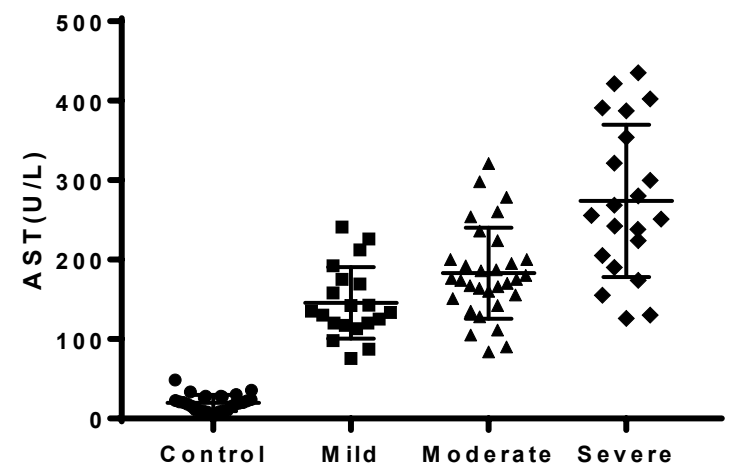

b

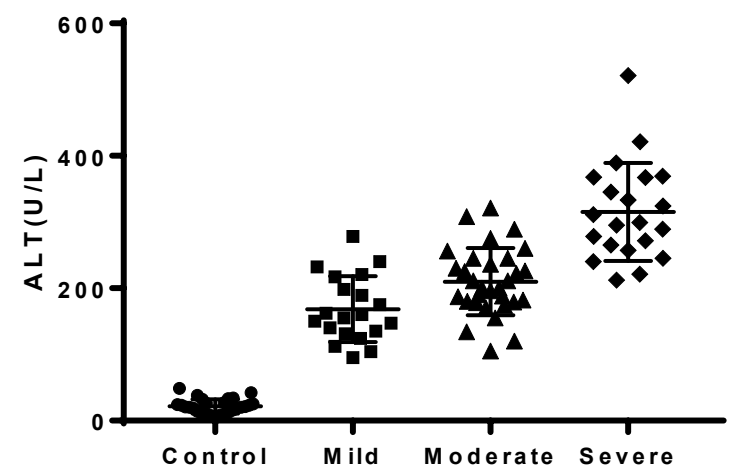

d

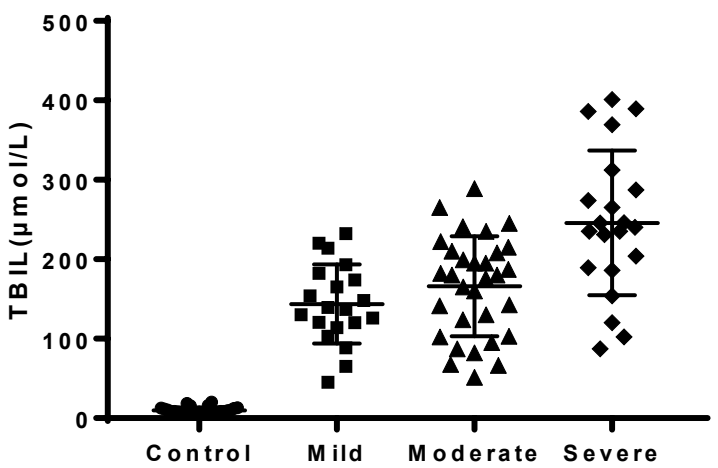

Figure 1: Scatter plot of serum Hcy, ALT, AST, TBIL and CHE in each group. 

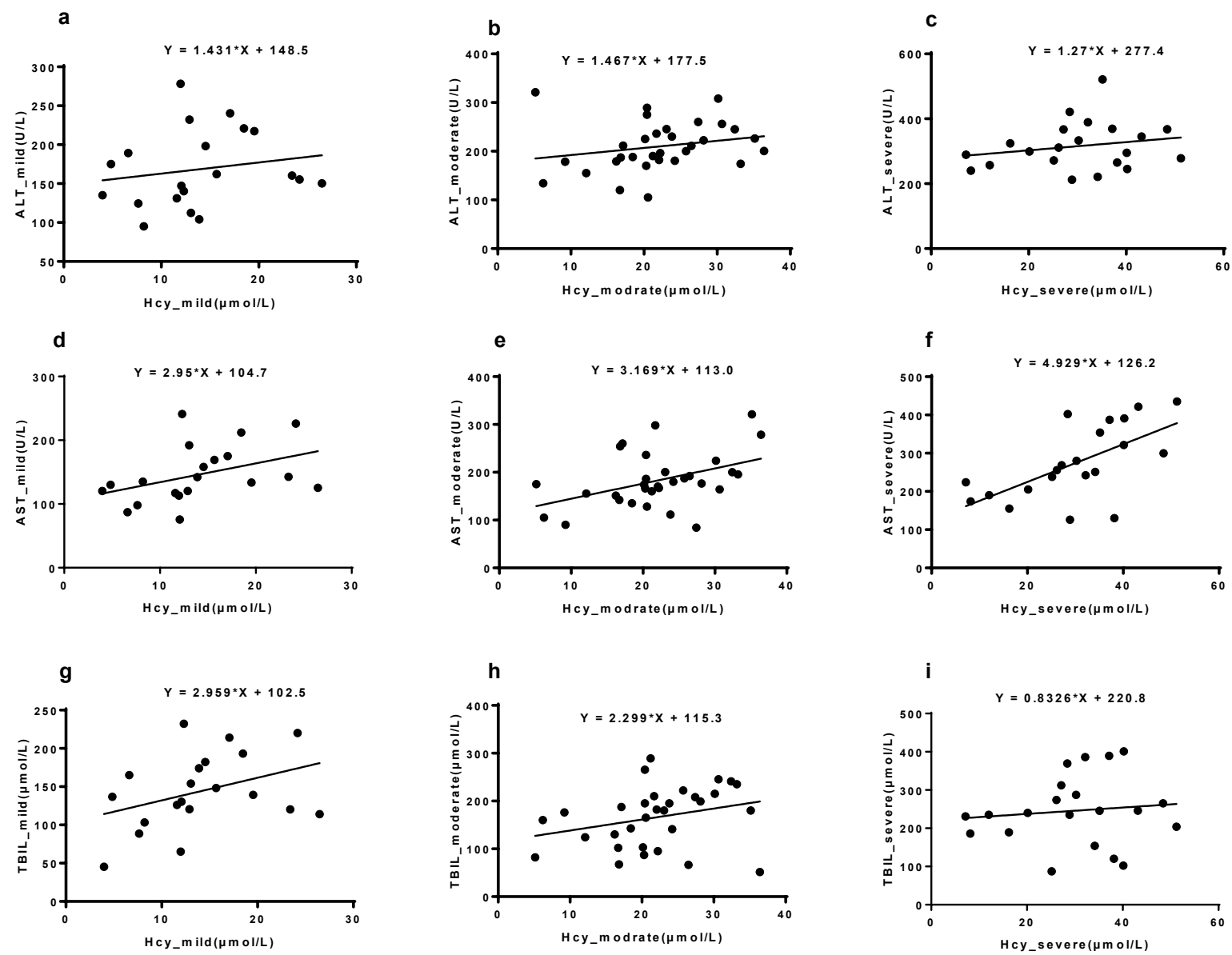

Figure 2: The Pearson correlation between Hcy and ALT, AST, TBIL in mild, moderate and severe disease groups.

liver diseases can affect Hcy metabolism and thereafter cause serum Hcy level changes. Several publications have evaluated the correlation of serum Hcy and HBV infection[15, 16]. Wang et al. [17] evaluated the correlation between Hcy and severity of hepatitis and found close correlation between the changes of Hcy and severity of hepatitis B. Zhu et al. [18] investigated serum Hcy as a prognostic biomarker for short-term mortality in HBVrelated acute-on-chronic liver failure patients and they found that serum Hcy level measured at admission may serve as a biomarker for 3-month mortality rate in patients with HBV-related acute-on-chronic liver failure. In this work, we evaluate the correlation between serum Hcy level and severity of chronic HBV hepatitis. We found that the serum Hcy level in healthy controls was significantly lower than that of hepatitis B patients. We also found that the more severe the hepatitis B the higher the serum Hcy was in hepatitis patients. This result indicated serum Hcy level in patients with hepatitis B reflected the degree of liver damage. Correlation analysis showed significant correlation between serum Hcy and AST in the moderate $(\mathrm{r}=0.43, \mathrm{p}<0.05)$ and severe disease groups $(\mathrm{r}=0.63, \mathrm{p}<0.05)$. However, the correlation between Hcy, and ALT, and Hcy and TBIL were not statistically significant in any group ( $p>0.05$ ). The correlation analysis demonstrated that the serum Hcy partly correlated with the serum transaminase level.

\section{In conclusion}

Hcy plays an important role in liver metabolism. Its serum level can reflect liver function, protein metabolism and hepatocyte damage. Therefore, the continuous increase of Hcy level can be regarded as a risk factor for the progression of hepatitis, and it can be used as serological marker for clinical diagnosis, treatment and prognosis. However, the results were limited because of the small sample size 
in the control and hepatitis B groups which need further approval by large-scale prospective multicenter clinical studies relevant to serum Hcy and hepatitis B.

Conflict of interest: Authors state no conflict of interest.

\section{References}

1. Li J, Zhang TY, Song LW, Qi X, Yu XP, Li FH, et al. Role of quantitative hepatitis $B$ core antibody levels in predicting significant liver inflammation in chronic hepatitis $B$ patients with normal or near-normal alanine aminotransferase levels. Hepatol Res. 2018;48(3):E133-E145.

2. Zhou J, Song L, Zhao H, Yan L, Ma A, Xie S, et al. Serum hepatitis $B$ core antibody as a biomarker of hepatic inflammation in chronic hepatitis B patients with normal alanine aminotransferase. Sci Rep. 2017;7(1):2747.

3. WHO Guidelines on Hepatitis B and C Testing. 2017. Geneva. World Health Organization.

4. Zhang Q, Liao Y, Chen J, Cai B, Su Z, Ying B, et al. Epidemiology study of HBV genotypes and antiviral drug resistance in multiethnic regions from Western China. Sci Rep. 2015;5:17413.

5. Li D, Long Y, Wang T, Xiao D, Zhang J, Guo Z, et al. Epidemiology of hepatitis $\mathrm{C}$ virus infection in highly endemic HBV areas in China. PLoS One, 2013;8(1):e54815.

6. Kumar A, Palfrey HA, Pathak R, Kadowitz PJ, Gettys TW, Murthy $\mathrm{SN}$. The metabolism and significance of homocysteine in nutrition and health. Nutr Metab (Lond). 2017;14:78.

7. Roblin X, Pofelski J, Zarski JP. [Steatosis, chronic hepatitis virus $C$ infection and homocysteine]. Gastroenterol Clin Biol. 2007;31(4):415-20.

8. Pastore A, Alisi A, di Giovamberardino G, Crudele A, Ceccarelli $\mathrm{S}$, Panera N, et al. Plasma levels of homocysteine and cysteine increased in pediatric NAFLD and strongly correlated with severity of liver damage. Int J Mol Sci. 2014;15(11):21202-14.

9. Sun W, Wei X, Zhao P, Niu A, Li C, Gao D. [Heparin-treated dendritic cells promote Th0 to Th1 differentiation via the Toll-like receptor 3 in peripheral blood monocytes of patients with chronic hepatitis B]. Zhonghua Gan Zang Bing Za Zhi. 2015;23(9):658-62.

10. Sabry R, ZAZ M, Abdallah AM. Relationship between Th1 and Th2 cytokine serum levels and immune response to Hepatitis B vaccination among Egyptian health care workers. J Immunoassay Immunochem. 2018;39(5):496-508.

11. Stelma F, Willemse SB, Erken R, de Niet A, Sinnige $M$, van Dort $\mathrm{K}$, et al. Dynamics of the Immune Response in Acute Hepatitis B Infection. Open Forum Infect Dis. 2017;4(4):ofx231.

12. Liu J, Wu H, Chen $\mathrm{H}$. Immune response to hepatitis $B$ vaccine in patients with chronic hepatitis $C$ infection: A systematic review and meta-analysis. Hepatol Res. 2018;48(2):119-126.

13. Boeijen LL, Hoogeveen RC, Boonstra A, Lauer GM. Hepatitis B virus infection and the immune response: The big questions. Best Pract Res Clin Gastroenterol. 2017;31(3):265-272.
14. Jiang HY, Wang SY, Deng M, Li YC, Ling ZX, Shao L, et al. Immune response to hepatitis $B$ vaccination among people with inflammatory bowel diseases: A systematic review and meta-analysis. Vaccine. 2017;35(20):2633-2641.

15. Zeng Dongxiao ZH, Huang Xin WQ. Investigation of the clinical value of serum homocysteine levels in patients with HBV related liver disease. Chinese Journal of Integrated Traditional and Western Medicine on Liver Diseases. 2016;(6):327-329.

16. GUO Ping HZHJLIJWJYM. The Amino Acid Metabolism and the Concentration Change of Homocystenine in Hepatitis B Patients. Journal of Kunming Medical University. 2011;(8):6265.

17. Ruifeng W, Juan Y. Study on the correlation between homocysteine and severity of hepatitis. Chinese Journal of New Clinical Medicine. 2017;(8):794-797.

18. [18] Zhu HD, Ma YC. Serum Homocysteine as a Prognostic Biomarker for Short-Term Mortality in HBV-Related Acute-onChronic Liver Failure Patients. Clin Lab. 2018;64(5):727-733. 